\title{
Evaluating spatial and frequency domain enhancement techniques on dental images to assist dental implant therapy
}

\author{
Shashikala J. ${ }^{1}$, Thangadurai $\mathbf{N}^{2}{ }^{2}$ \\ ${ }^{1}$ Department of Electronics and Communication, JAIN (Deemed-to-be University), BMS Institute of Technology and \\ Management, Bengaluru, Karnataka, India \\ ${ }^{2}$ Department of Electronics and Communication, Faculty of Engineering and Technology, Centre for Emerging \\ Technologies, JAIN (Deemed-to-be University), Bengaluru, Karnataka, India
}

\begin{tabular}{l}
\hline \hline Article Info \\
\hline Article history: \\
Received Jan 4, 2021 \\
Revised Apr 8, 2021 \\
Accepted Apr 27, 2021
\end{tabular}

Keywords:

CBCT

Contrast enhancement

Dental x-ray

Optimization

Preprocessing

\begin{abstract}
Dental imaging provides the patient's anatomical details for the dental implant based on the maxillofacial structure and the two-dimensional geometric projection, helping clinical experts decide whether the implant surgery is suitable for a particular patient. Dental images often suffer from problems associated with random noise and low contrast factors, which need effective preprocessing operations. However, each enhancement technique comes with some advantages and limitations. Therefore, choosing a suitable image enhancement method always a difficult task. In this paper, a universal framework is proposed that integrates the functionality of various enhancement mechanisms so that dentists can select a suitable method of their own choice to improve the quality of dental image for the implant procedure. The proposed framework evaluates the effectiveness of both frequency domain enhancement and spatial domain enhancement techniques on dental images. The selection of the best enhancement method further depends on the output image perceptibility responses, peak signal-to-noise ratio (PSNR), and sharpness. The proposed framework offers a flexible and scalable approach to the dental expert to perform enhancement of a dental image according to visual image features and different enhancement requirements.
\end{abstract}

This is an open access article under the CC BY-SA license.

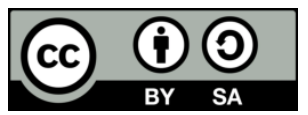

\section{Corresponding Author:}

Shashikala J.

Department of Electronics and Communication

JAIN (Deemed-to-be University)

BMS Institute of Technology and Management, Bengaluru, Karnataka, India

Email: shashikala_j@bmsit.in

\section{INTRODUCTION}

Dental implant surgery often refers to a complicated subject that requires an extreme level of skill and proficiency in a wide range of clinical dentistry disciplines. The entire implantation process is dynamic, and a good healing environment is not always possible to achieve. Complications inevitably occur; quantifiable measures can be useful in preventing such complications. These include inclusive clinical assessments and radiographic analysis to predict possible complications and treat accordingly. However, few of these problems need little clinical care, while some may demand a comprehensive pre-operative assessment by specialists in different dentistry disciplines. The advancement into image acquisition technology and radiology benefits various modalities of human organ diagnosis such as bone disorder analysis, nerve disorder, organ malfunctioning, and growth of abnormal tissues, facilitating the various stages of treatment planning for the clinical studies [1]. The radiology used for the dental diagnosis and treatment is 
popularly known as a dental radiograph, imaging dentistry, or oral and maxillofacial radiology (OMFR) [2]. There are various forms of dental imaging modalities. However, the dental $\mathrm{x}$-ray image is quite popular, and another imaging modality cone-beam computed tomography (CBCT) is gaining popularity due to its advanced feature of visualizing the maxillofacial region from different views. CBCT provides interactive exhibition methods such as multiplanar reconstruction and 3-dimensional evaluation, which helps to visualize an affected tooth's position associated with surrounding critical structures and adjacent teeth. In order to avoid any ill-effect, the radiation is kept low while taking the dental x-ray [3]. The dental x-ray produced at low radiation and limited capacity devices provides very low-quality images with poor contrast and brightness, causing visibility differences and ambiguity while analysis [4]. The contrast of the dental image is degraded due to the penetrating power of the radiation source, thickness difference, and Density difference [5]. The specific noises and artifacts due to patient movement, including breathing and heartbeat during the radiography, because imaging artifacts to the dental image. The dentist or maxillofacial surgeons must know more specific details such as lamina dura, dentin-thickness, pulp tissue conditions for filling, better analysis, treatment, and surgical implanting plans [6]. Therefore, enhancement techniques are essential for dental images to provide visually analyzable input to dental professionals [7].

In dental images enhancement, the prime objective is to give more visibility to the core information and neglect or nullify the secondary features or information to improve the quality of visual perception [8], [9]. Both the objective of the perception by the human vision system (HVS) and computer vision system (CVS) shall meet the respective goal of diagnosis by humans and computers. The visual characteristic of the dental images varies due to pixel differences in grayscale across the spatial domain. The gamma correction method (GCM) is applicable in such cases [10]. The GCM of image enhancement for dental images is evaluated in the proposed framework [11]. Another popular method is histogram-based histogram equalization and contrast limited adaptive histogram equalization (CLAHE) [12], [13]. These techniques are widely accepted in medical image enhancement due to the frequency distribution of visual statistics from the histogram. Apart from this, there are many other enhancement techniques used in the enhancement of images. However, each technique is associated with some advantages and limitations [14], [15]. Another factor is selecting an appropriate enhancement mechanism is very difficult, as the choice depends on the image of interest and treatment objectives. Therefore, the proposed study introduces a framework that automates the selection of enhancement techniques based on the dental image preprocessing requirement. The significant contributions of the proposed study are being as:

- The proposed study evaluates different spatial and frequency domain enhancement techniques for selecting improved dental images based on enhanced image visual quality.

- The study presents the modeling of a universal framework that integrates the functionality of various enhancement mechanisms with variable user choice options so that dentists can select based on their requirements.

- The design of the proposed framework is flexible and scalable that can support other imaging modalities or domains to perform image enhancement.

- The proposed framework offers a cost-effectiveness and quality approach in clinical analysis towards carrying out pre-operative assessments to advantage the patient screening process.

- The proposed framework has also incorporated the optimization functionality in the dental image enhancement to analyze better images based on particular requirements.

- The selection of a suitable enhancement mechanism for a particular dental image will depend on the visual image feature. The selection of enhanced image depends on the outcome, i.e., human eye evidence (visual quality) and the output image statistics in terms of peak signal to noise ratio (PSNR), sharpness.

The rest of the sections in this paper are organized in the following manner: Section 1.1 presents the background and research problem in section 1.2. Section 2 discusses the proposed system and its implementation design. Section 3 highlights the result and outcome analysis. The conclusion of the overall work presented in this chapter is mentioned in section 4.

Over the last decades, enormous research has been carried out towards medical imaging preprocessing and image quality enhancement. This research study considers a case study of the enhancement of dental imaging to benefit implant therapy. This section presents related work in the context of dental x-ray image and CBCT image enhancement. The work done by Choi et al. [16] focuses on the multi-level enhancement of the periapical dental image. The authors first used histogram techniques to maintain even distribution and perform normalization of the input image, affected by low doses used during patient imaging. On the other hand, the contrast limited adaptive histogram contras limited adaptive histogram equalization (CLAHE) is adopted to improvise local contrast of the image, carried out with a different level of contrast adjustments. Further, the sharpness of the image is maintained using an un sharp masking mechanism. Kamezawa et al. [17] considered the case of the CBCT image captured with a low dose 
and image-guided patient positioning system. The noise introduced in the CBCT image due to patient positioning setup and low dose CBCT was suppressed using a denoising approach. Various denoising mechanism was used in this study to evaluate the performance of each denoising methods towards identifying suitable denoised image. The study outcome demonstrates that the edge-preserving denoising technique achieves higher noise reduction. Yin et al. [18] presented a thresholding mechanism-based noise suppression algorithm for CBCT image enhancement. Thresholding is applied in the wavelet coefficient magnitude divided into two different parts as irregular and regular coefficients. Based on the CBCTprojection noise adjustment estimation technique is designed to perform denoising operations. The outcome exhibits an increased rate of PSNR achieved by the introduced mechanism.

Amiri et al. [19] conducted a study focused on positioning error in a panoramic dental image. The study highlighted that due to positioning error, the imaging processes are carried out recursively. Patients have to be exposed to radiation every time, which is a significant concern to patient health. The authors suggested enhancement over panoramic images to avoid repetitive process imaging. In this study, an automatic positioning error correction model is introduced with an adaptive GCM to enhance the image to highlight the maxilla sinusoid region. Another research work towards enhancing the panoramic dental image is carried out by Kandan et al. [20]. In this, the authors have used a combined approach of GCM and a Gray level co-occurrence matrix to address poor contrast and luminance nonlinearity. The combined approach was used to reduce the homogeneity factors, where the GCM values are globally defined based on the cumulative histogram. The quantitative analysis in terms of PSNR and mean square error (MSE) demonstrates better image enhancement by the presented approach. The histogram equalization usage is seen in the study of Naik et al. [21], where the authors have focused on the problem of sameness of the foreground and background of the dental image. The study aimed to evaluate the performance of histograms against poor visibility and under and over-exposed dental images.

Qassim et al. [22] evaluated different enhancement techniques in their study to improve dental x-ray periapical images. The authors have considered log transform GCM and different histogram-based techniques. The performance evaluation of each technique was conducted for a particular image. The outcome suggested that CLAHE is the most suitable technique for preserving brightness in the dental images under analysis. However, enhancement technique efficiency is justified based on the visual outcome and less on the quantified outcome. The usage of single-scale retinex based image enhancement is also found in the study of Dai and Zhang [23]. The authors have improvised the function of a single retinex mechanism, and based on the histogram analysis, the dental image was enhanced. In order to reduce noise effects in the enhanced image, a denoising function of the weighted coefficient mechanism is introduced. Georgieva et al. [24] adopted an approach of CLAHE and mathematical morphological operation to detect caries in the dental image. On the other hand, the authors have applied a homomorphic denoising technique to address noisy signals and uneven distribution of the luminosity factor. Enhancement of the CBCT image is done in the study of Khattar et al. [25] to highlight the significant features to assist endodontic therapy. An enhancement approach based on the multi-scale-retinex is applied to achieve an enhanced image. The performance of the presented technique is assessed against CLAHE and histogram equalization (HE). The outcome suggested thatmulti-scale-retinex can be efficiently applied to enhance the digital image. However, this approach may require a long run time in the enhancement execution process due to the inherent complexity of the multiscale-retinex mechanism. Momoh and Omuya [26] used several enhancement methods such as CLAHE, contrast stretching, contourlet transform, and wavelet transform to reduce noise and enhance the image contrast factor. In the evaluation, CLAHE achieves better performance in terms of MSE and PSNR.

Based on the review analysis, a significant research problem is a highlight is being as:

- The existing research studies towards medical imaging enhancement are extensive. However, very few works have been presented on dental imaging enhancement.

- Dental image enhancement techniques are mostly carried in the limited scope of preprocessing operation, which may not meet the requirement enhancements needed to address multiple issues.

- Few of the existing dental image preprocessing mechanisms are also subjected to longer run time.

- There is a minimal method that can provide better enhancement over low contrast and dark dental images.

Therefore, the problem statement of the proposed study can be expressed as "designing a costeffective framework is a challenging task that can offer a single-handed preprocessing computation to address multiple problems of dental image quality where enhancement is carried out based on the evaluation."

\section{PROPOSED FRAMEWORK}

This section provides a detailed description of the methodologies and implementation strategy adopted to determine the best suitable enhancement operation over the dental image towards achieving a 
higher success rate in implant therapy. Medical image contrast adjustments and brightness preservation improve the visual aspects and highlight the region of an image under consideration. To enhance contrast and adjust un-even illumination in dental images, this paper proposes modeling of an efficient computational framework for assessing frequency and spatial domain enhancement mechanisms for a given input dental image. The selection of appropriate enhancements depends on the response of the preprocessed image. In this regard, the evaluation of image statistics (brightness, sharpness) and performance metrics (PSNR, MSE) is carried out for each preprocessing technique applied to a particular input image. The contribution of the proposed work is also to facilitate physicians with a flexible approach that meets all enhancement requirements needed to improve dental imaging quality towards the effective patient diagnosis and preoperative assessment for a successful implant. The block diagram of the proposed computational framework is shown in Figure 1.

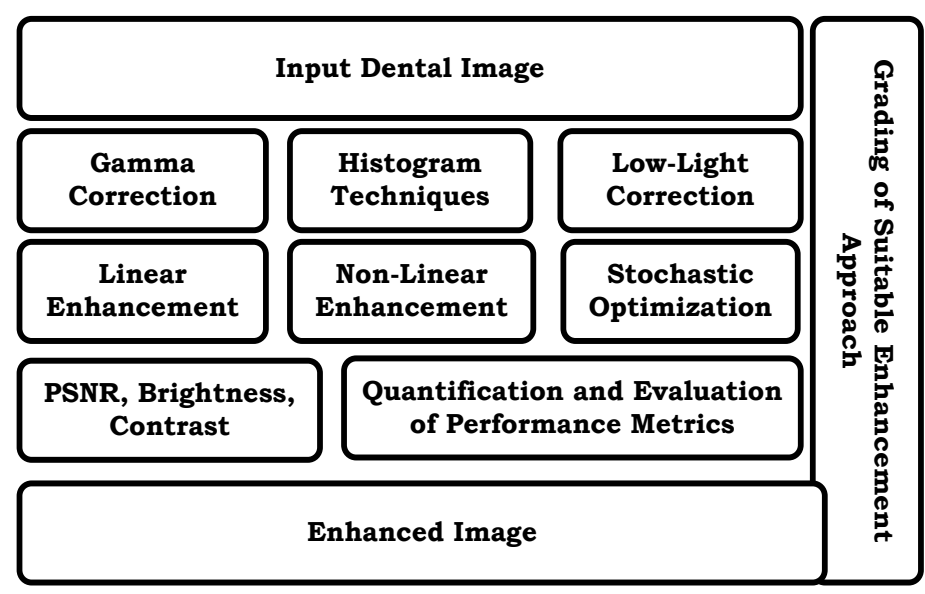

Figure 1. Shows a schematic outline of the proposed system representing the flow of input dental image processed and evaluated with different enhancement operation

The input dental images are typically subjected to various quality issues, including poor resolution in the spatial domain, low contrast, imaging artifacts (misrepresentations of tissue), and a high noise level. These visual quality issues may be inherent to the imaging modalities, resulting from the lightening condition, image capturing environment, or the patient movement during acquisition. In this paper, multiple algorithms, namely GCM, histogram equalization, CLAHE swarm optimization-based enhancement, linear and nonlinear enhancement, and lowlight image correction, are explored and evaluated towards identifying their effectiveness to address various visuals quality issues in dental imaging. In this paper, the study is only interested in designing an adaptive multi-functional interface model to assist the clinical analysis for a better outcome for patient diagnosis and treatment and their health well-being. Therefore, the proposed system presents a unified framework that integrates various preprocessing functions to address image quality imperfections based on the different enhancement requirements.

\subsection{Implementations of preprocessing techniques}

This section presents the implementation strategy adopted in the proposed system, followed by algorithmic steps and discussion.

\subsubsection{Gamma correction}

Since the dental images contain the texture of maxillofacial objects such as jaws and teeth, the medical imaging modalities use a mapping function known as power-law-transformation for capturing and displaying images. However, a distortion called gamma falsification often occurs in the output images due to the technical problems associated with imaging modalities. This has a nonlinear tristimulus effect on each pixel of the image, causing an output image with low contrast and uneven illuminance. Therefore, the proposed framework for enhancing dental imaging $(\mathrm{dIm})$ includes the gramma correction technique. In the GCM, the pixel value is controlled according to the relationship between the pixel data and the GCM $(\gamma)$ value to highlight the hidden details in the brighter and dark area to the HVS. Fundamentally, the input to the output mapping of the pixel (p) is mapped as $f \gamma(p) \rightarrow f^{\prime}(p)$ such that the GCM function (1). 


$$
(\mathrm{dIE})=255 \times(\mathrm{dI}(\rho) / 255)^{1 / \gamma}
$$

Where, $\mathrm{f} \gamma(\mathrm{)}) \leftarrow \mathrm{GCM}$ function, $\gamma \leftarrow \mathrm{GCM}$ correction factor, $\mathrm{dIE} \leftarrow \mathrm{GCM}$ corrected dental image and $\mathrm{dI}(\rho) \leftarrow$ input image pixel $\mathrm{p}$. The proposed framework provisions access to digitize the raw dental image with lower visual perception, and further, the enhancement is applied for the different values of $\gamma$ set $\gamma \leftarrow 0.1: 1$.

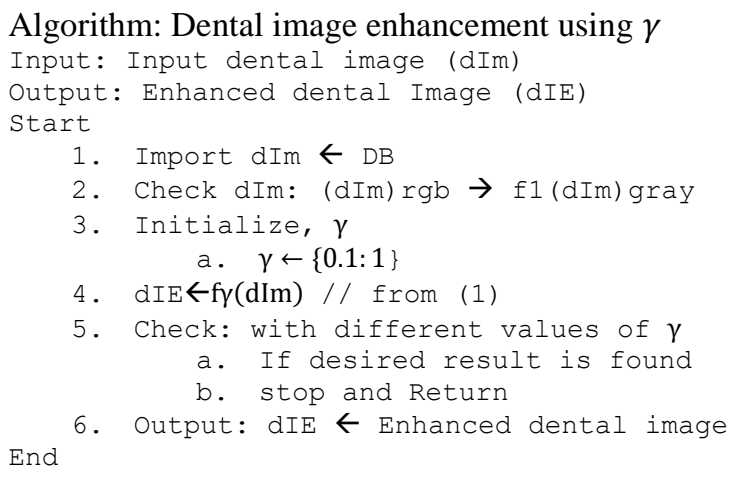

The algorithm takes input as a dental image $\mathrm{dIm}$, and after applying GCM, it provides contrastadjusted image dIE. The system imports the dIm from the database and checks the dimension size to convert it into a grayscale image (Line 1,2). The conversion of red, green, dan blue (RGB) dental image to grayscale provides computational efficiency while applying preprocessing over the input image. In the next step, a variable $\gamma$ as a correction factor is initialized. The proposed system considers its values in the range of $0.1: 1$ to evaluate a particular input image with varying values to achieve a suitable enhanced image (Line 3 ). The computation of enhanced image with correction factor $\gamma$ is computed using (1) (Line 4). The previous step (Line 4) will be repeated with different $\gamma$ values to evaluate the enhanced image quality.

\subsubsection{Histogram techniques}

Histogram-based techniques have been widely used in medical image enhancement in the literature. The histogram of the dental image represents the probability distribution of gray values and shows that the image contrast is low, dark, or distributed and considerable. The relationship between the histogram and the visual quality of the image is shown in (2) is being as:

$$
\left\{\begin{array}{cc}
\text { Histogram Weighted to Right } & \text { Bright } \\
\text { Hitogram weighted to left } & \text { Dark } \\
\text { Distributed } & \text { Good quality }
\end{array}\right.
$$

The visualization of the histogram of an input image provides an analysis of the frequency distribution of visual statistics in the image. The histogram of any image is a plot function of hist (ri) and intensity levels (ni $\in I$ ). The study considers the evaluation of two different histogram-based techniques for image enhancement discussed in respective sub-sections.

$$
\operatorname{histp}\left(r_{i}\right)=\frac{n_{i}}{m x n} \text { where } i=0,1,2 \ldots I
$$

\subsection{Histogram equalization}

Histogram equalization (HE) generates images with possibly the same intensity level in the entire image. The equalization intensity level generates an image with an up-scaled dynamic range, resulting in increased contrast. In this process, a transformation mapping function $\mathrm{T} f()$ expands the image intensity values within the range $0: 1$, leading to a contrast-enhanced image. The HE transformation function $\mathrm{T} f()$ refers to cumulative-distribution described is being as:

$$
\begin{aligned}
& c d f(I)=\sum_{i=0}^{I} h i(n i): I \\
& T f()=\left\lfloor\frac{I-1}{m x n} \sum_{i=0}^{I} k i\right\rfloor
\end{aligned}
$$


The computational process includes estimating the number of pixels intensity and the probability of each pixel intensity in the input image. The algorithm also calculates the cumulative distribution function (CDF) and then multiplies it by the range defined for the processed image. In the next step, the algorithm uses the rounding function to map the obtained pixel values towards the lowest integer values, which turns out in the higher pixel intensity value in an image with higher contrast.

\subsection{CLAHE}

CLAHE is another technique based on histograms introduced as an advanced version of HE. CLAHE overcomes the over-brightness problem caused by HE and produces good results on dental images. The steps involved in CLAHE-based dental image enhancement are discussed is being as:

1. Divide the input dental image into even sizes $\mathrm{M} \times \mathrm{N}$ and non-overlapping blocks.

2. Compute the histogram for each block.

3. Compute contrast-limited histogram for each block.

4. Compute mean pixels.

- Nmean $=($ dIx $x d I y) / g$, where, $\mathrm{dIx}$, dIy is the dental image pixel dimension, $\mathrm{g}$ is the gray levels.

5. Limit for contrast expansion $\mathrm{CE}$ is given, and CL is the actual clip limit, such that $C L=\frac{M}{N}(1+$ $\left.\frac{\varphi}{100}(C E-1)\right)$, where $\mathrm{M}, \mathrm{N}$ are the pixels and gray level in each block, respectively. The attribute $\varphi$ refers to the clip limit factor, and CE is the contrast expansion limit used by the transformation function. The attribute $\varphi$ ranges between 0:100, the histogram slope in each mapping process will be (1-CE).

6. Clip the histogram and then use the clipped histograms to calculate the CDF.

7. Improve Iv (intensity values) using distribution transform Rayleigh given by: $r(i)=\mathrm{lb}+$ $\sqrt{2 \alpha^{2}\left(\frac{1}{1-C D F}\right)}$

8. Based on the Iv of that pixels, find its mapping based on their CDF value.

9. Eliminate unwilling artifacts at boundary via bilinear interpolation.

\subsection{Image scaling}

The proposed also incorporates the benefits of the image scaling processes in the proposed framework as shown in the Figure 2. The prime advantage of this technique is that the input image can be resized based on the computational requirement and memory space. There are many methods available for image scaling. Among them, interpolation based on nearest-neighbor is simple and has a faster frequency response. Dental image (dI) scaling using nearest-neighbor interpolation can be given is being as:

$$
d I^{\prime}=f_{S}(d I, r r, c r)=f_{S r}\left(S_{c}(d I, c r) r r\right)=f_{S c}\left(S_{r}(d I, r r) c r\right)
$$

Where, $d I^{\prime}$ indicated scaled dental image, $f_{S}$ refers to the scaling function, $d I$ original input dental image, $r r$ is the horizontal (row-wise) scaling ratio, and $c r$ is vertical (column-wise) scaling ratio. The function $f_{S r}($ ) and $f_{S C}()$ is the scaling function with respect to row and column.

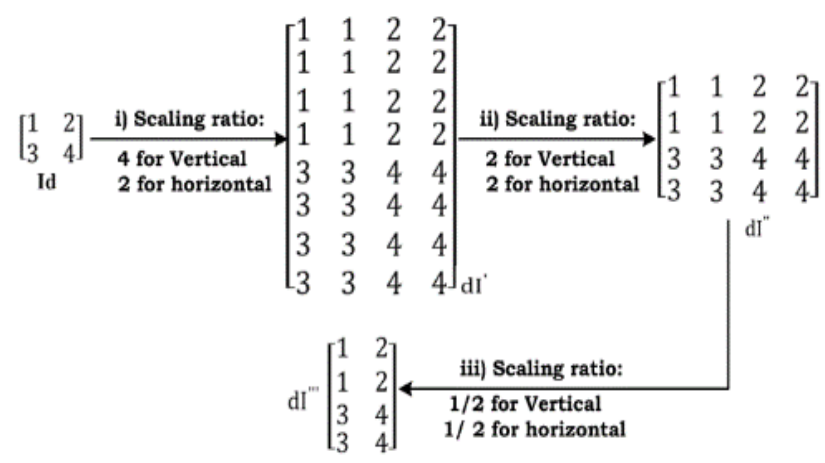

Figure 2. Shows a generic process of image scaling

The significant steps involved in this process are given with a simple example is being as:

1. Imp

2. ort input image to the system: $d I$ 
3. Compute the size of input image: $[r, c] \leftarrow d I$

4. Define new size for interpolation to get output image: $[n r, n c] \leftarrow d I^{\prime}$

5. Compute the ratio of new size with input image size:

6. $r r \leftarrow n r / r$

7. $r c \leftarrow n c / c$

8. Compute interpolated position:

9. Row-wise: $I r \leftarrow f_{\text {round }}((r \times r r) / r r)$

10. Column-wise: $I c \leftarrow f_{\text {round }}((c \times r c) / r c)$

11. Construct a matrix of zeros: [ ] $n r \times n c$

12. Interpolate: $[I r, I c] \rightarrow[]_{n r \times n c}$

13. Interpolated matrix $\leftarrow d I^{\prime}$

The advantage of image scaling is that it can maintain the uniform size of all input dental images in the dataset to assess the linear performance of the automated computational model for pre-operative assessments in the dental implant.

\subsection{Evaluation of linear and non-linear enhancement techniques}

The proposed study introduces the implementation of linear and nonlinear enhancement techniques in the proposed framework to address high level and random noise in the image at frequency levels.

\subsubsection{Linear enhancement}

Linear enhancement is used to address the quality of dental images degraded during the image acquisition environment. In this enhancement process, the linear enhancement reduces the impact of random noise and enhances the contrast of the images. The algorithm for dental image enhancement using a linear enhancement is given is being as:

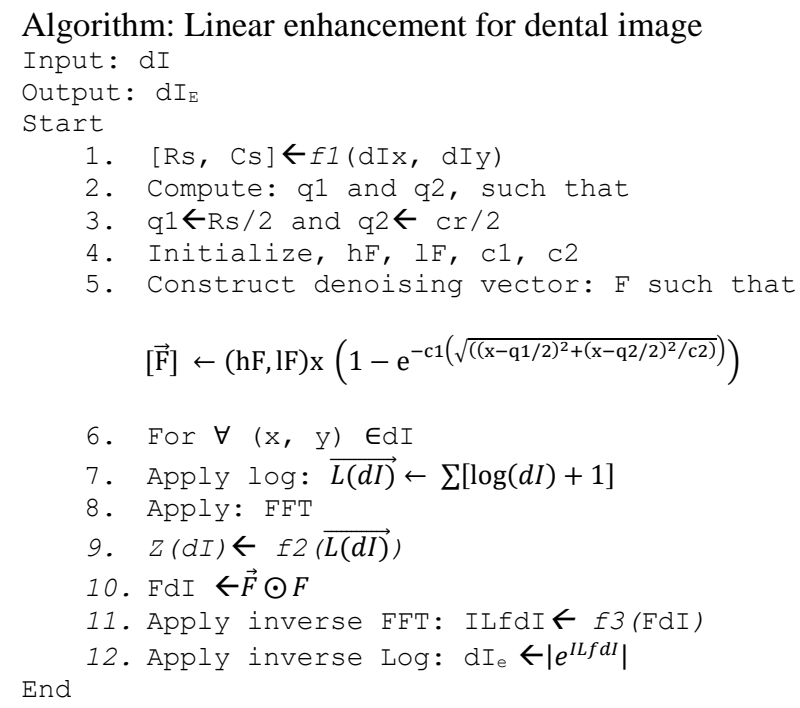

This algorithm performs enhancement operations on the dental image $\mathrm{dI}$ in-frequency domain. The system takes input as variable $\mathrm{dI}$ and computes the size as row 'Rs" and the column "Cs" using function $f 1()$ with an input argument of dental image dI (Line 1). The algorithm then computes q1 and q2 as the centroidal component for the denoising vector construction (Line 2, 3). The system initializes four different variables $\mathrm{hF}$ (high frequency co-efficient), $\mathrm{IF}$ (low-frequency co-efficient), $\{\mathrm{c} 1, \mathrm{c} 2\}$ frequency adjustment coefficients (Line 4). Further, for each pixel $\mathrm{x}$, y of $\mathrm{dI}$ denoising vector constructs takes place $[\vec{F}]_{m, n}$ (line 5,6$)$. In order to perform a linear operation in the frequency domain, logarithmic of input $\mathrm{dI}$ is computed using function $f 2$ (), and further fast Fourier transformation FFT takes place to perform enhancement operation of dI (line 69). The enhancement operation is then carried out based on the dot product of vector $\mathrm{F}$ and $\mathrm{Z}$ (dI) input dI infrequency domain. Further, to achieve denoised image FdI in its original domain, inverse fast fourier transform (FFT) using function $\mathrm{f} 3$ and inverse logarithmic operation is carried sequentially (Line 11, 12), which provides final output as dIE, i.e., linear enhanced image. The advantage of using a linear enhancement operation is that it yields better improvement over input image subjected to random noise, and also, it overcomes the issue of over brightness.

Evaluating spatial and frequency domain enhancement techniques on dental images to ... (Shashikala J.) 


\subsubsection{Indirect enhancement}

A nonlinear enhancement operation is constructed to address poor image quality due to high-level noise and lack of smoothing effect in the edges of input dI. This study uses a nonlinear enhancement operation on the input $\mathrm{dI}$ as a logarithmic single-channel intensity image. The algorithmic steps of nonlinear enhancement are carried out is being as:

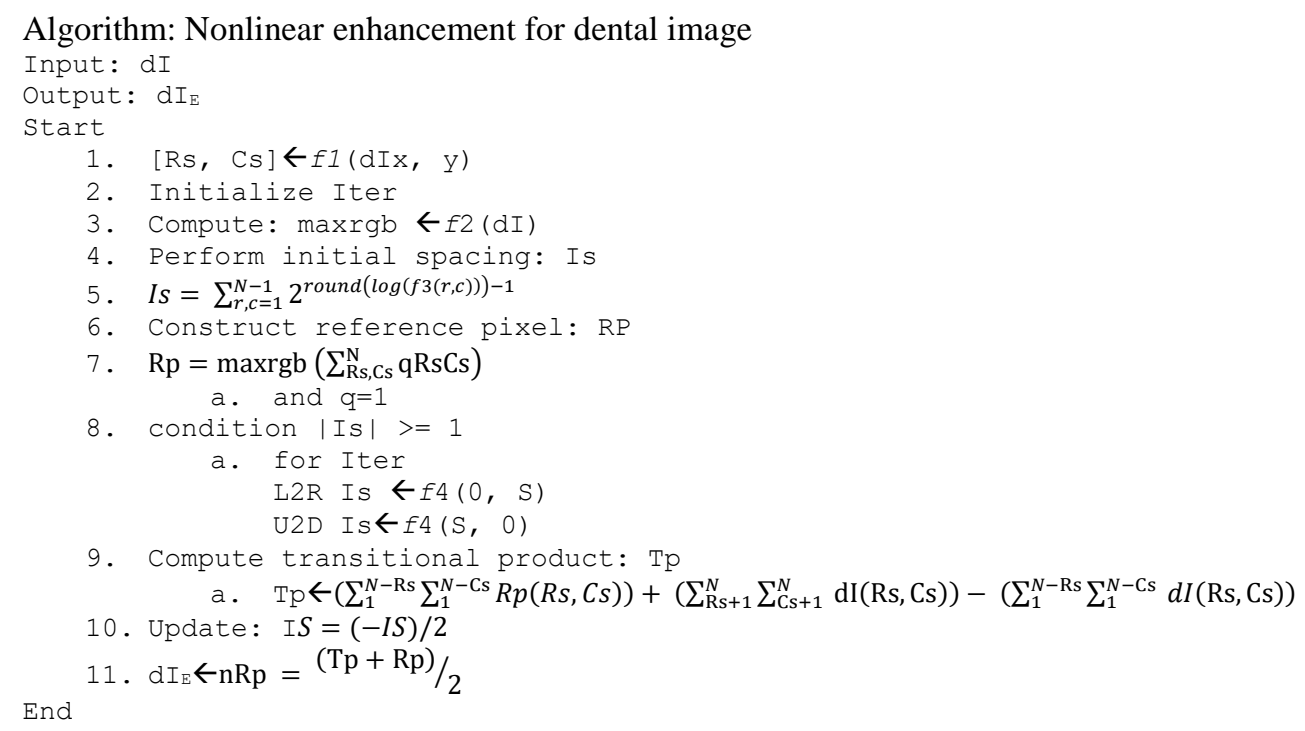

The algorithm computes the size of the input image using function $f 1()$ and provides row size "Rs' and column size 'Cs' respectively (Line-1). In the next step, a variable Iter (number of iterations) is initialized to compute an enhanced image until a better output image is achieved (Line 2). An explicit function $f 2()$ is applied over dI to store maximum color value 'maxrgb' of dI to construct a reference image having the same resolution size of an input image dI (Line-3). In the next step, the initial spacing between each pixel of dI gets computed based on which the enhancement operation is carried out (Line 5,6). The computation of reference pixel ( $\mathrm{Rp}$ ) is shown in algorithm Line-7. In this process, a reference matrix is created with maxrgb and maintains its size concerning Rs and Cs of dI.

Further, a condition is checked for the initialized Iter to perform enhancement operation. In this process, a spacing count is carried out to eliminate redundant pixels. This process involves horizontal spacing count-(left-to-right L2R) and vertical spacing count (U2D up-to-down). This process performs mapping of the linear distribution of pixel over nonlinear pixel (Line-8) and eliminates the non-specific pixel values as well as maintains the even distribution of illumination based on the spacing count between each pixel of the dI. In the next step of the algorithm, the system computes a transitional product (TP) to construct a new reference pixel matrix from the outcome obtained after the spacing count in the previous step (Line-10). The value of initial spacing gets updated (Line-11), and the outcome electronic data interchange, (EdI) gets computed based on the computation of new reference pixel (nRp) followed by the value obtained for take profit (TP) and roleplaying (RP) (Line 12).

\subsection{Evaluation of lowlight image enhancement}

The dental images captured in a low-lightening environment are subjected to low visibility, poor contrast, and dynamic noise, which degrades diagnosis by HVS and affects the computational performance in advertising solution (ADS). The study introduces lowlight dental image enhancement based on an application of haze removal algorithm and edge-preserving function for noise removal to achieve a better quality of the enhanced dental image.

The system takes input dental image dI gets a complement of dI. The complement of the image is computed based on the numerical operation where the input image $\mathrm{dI}$ is subtracted from the maximum color value of the image (Line-1). In the second step of the algorithm, a function f1() is applied to perform enhancement operations using a haze removal algorithm (Line-2). The above two steps perform a primary enhancement. In order to compute a more enhanced version of input image dI, the algorithm performs a similar operation of complement and enhancement using function f1 () over H1 primary enhanced image (Line-2 to 5).

Further, the secondary enhanced dental image $\mathrm{H} 2$ is then subjected to another function, f2(), which performs a smoothing operation. As a result, it preserves detailed edge information in the final enhanced 
dental image IdE (Line-6). This operation reduces the impact of the lowlighting condition on the image. It provides better visibility to the input dI image, highlighting objects with the difference in the image background and foreground view.

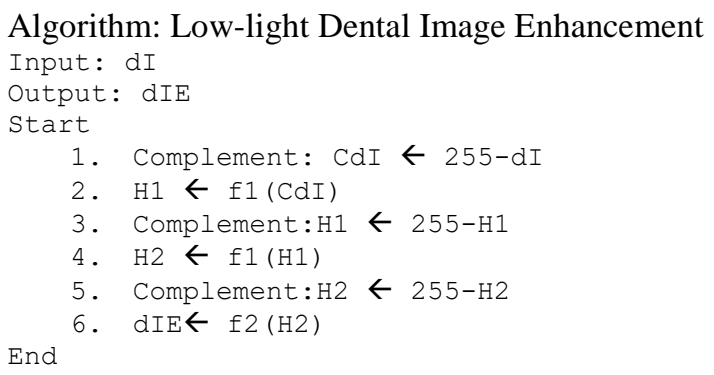

\subsection{Evaluation of optimization in enhancement}

The proposed system also incorporates the application of a nature-inspired optimization algorithm to perform optimized enhancement on input dental images. The study considers an enhancement approach based on swarm-based optimization. The algorithmic steps in the implementation of optimization in the enhancement process are described is being as:

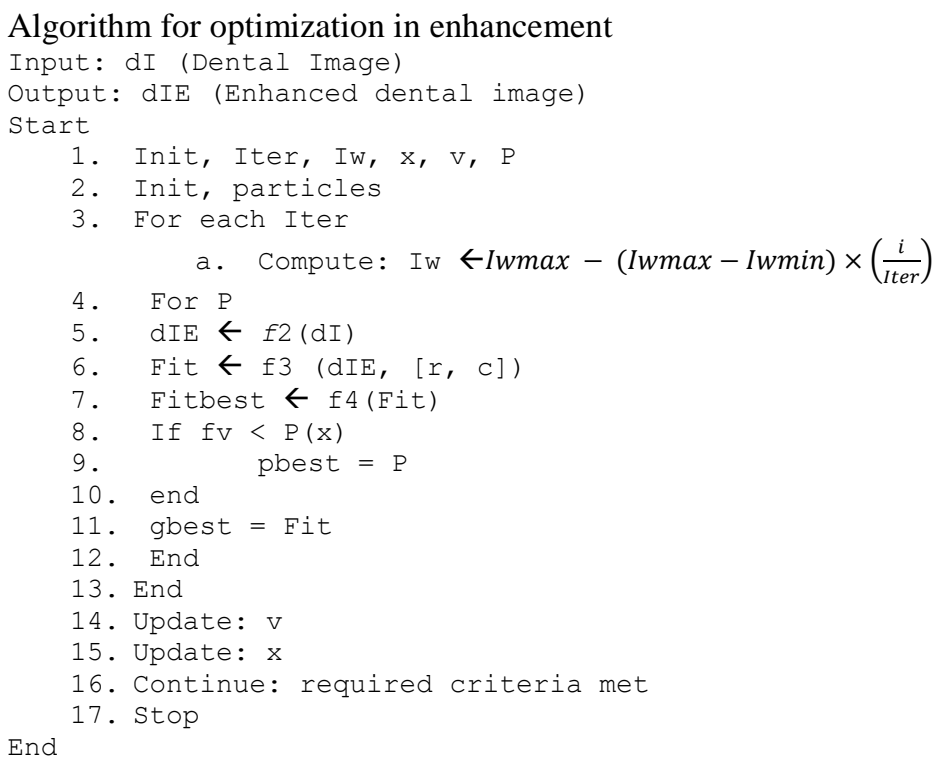

The algorithm takes input value as $\mathrm{dI}$ and generates enhanced dental image (EdI) after execution with an optimization algorithm. In the first step, the initializes the solution space variables as max-iteration (Iter), inertial weight (Iw), particle position (x), particle velocity (v), particle size (P) (Line 1). The algorithm initializes particles for given Iter to compute an enhanced image (Line 2). For each Iter, computation of Iw is carried out to determine the optimal set for parameters initialized. It also means that the parameters are subjected to each particle or solution (Line 3). For each particle size, the algorithm computes enhanced dI using function $\mathrm{f} 2($ ) with an input argument of $\mathrm{dI}$, and initial particle position (Line-5). Here the function fx2() refers to a transformation operation to enhance the input image. In the next step of the algorithm, discrete function $\mathrm{f3}()$ with an input argument of the enhanced image and size of an image $(\mathrm{m}, \mathrm{n})$ is considered for computing fitness value Fit (Line-6). The further maximum value in Fitmax gets computed (Line 7). The gbest is computed as the maximum value of the Pbest (Line 9-11), and updating of particle new position $\mathrm{x}$ and velocity $\mathrm{v}$ is continued till the required criteria are matched (Line14 to 17).

\section{RESULTS AND DISCUSSION}

The implementation of the proposed model is carried on a numerical computing tool MatLab. This section presents the result of the proposed system and discusses the performance exhibited by different enhancement techniques. The performance evaluation is carried out for two different dental x-ray images. The first input image is a dental x-ray, and the second one is a dental CBCT image. The performance for each 
technique is analyzed concerning qualitative analysis and quantitative analysis. In the qualitative analysis, the visual outcome and histograms are presented for human perception or human visual analysis. The quantitative analysis is carried out in terms of the PSNR and sharpness of the output image. The study considers two types of dental images. The first one is a dental x-ray, and the second one is CBCT. The visual outcome is tabulated in Table 1 and Table 2.

Table 1. Visual analysis

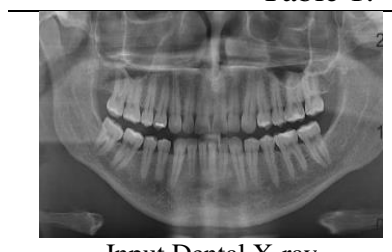

Input Dental X-ray

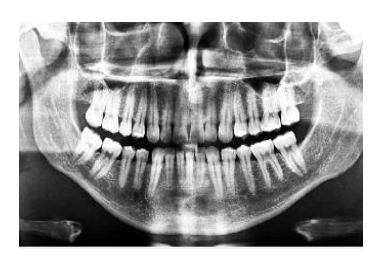

GCM

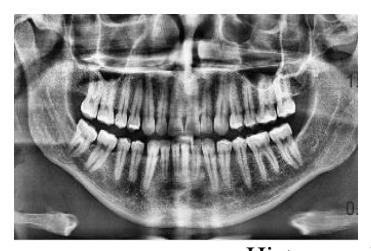

Histogram Equalized Image

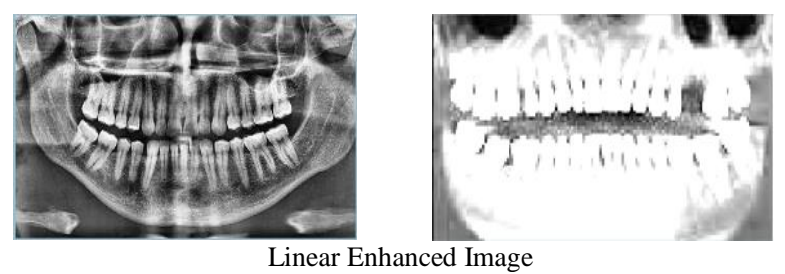

Linear Enhanced Image

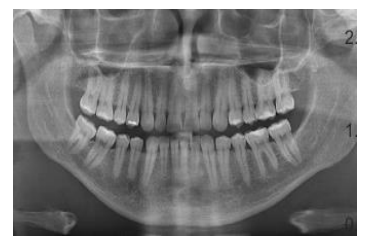

Non-Linear Enhanced Image
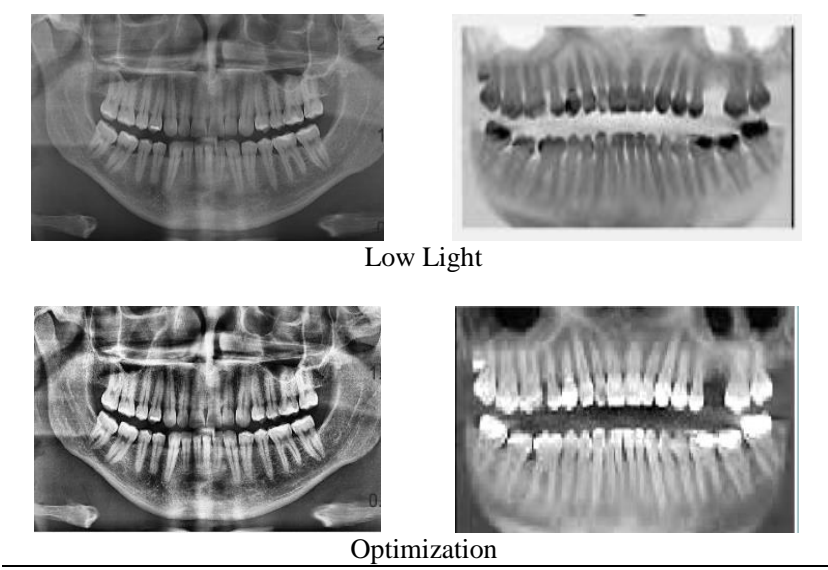
Table 2. Analysis of histogram
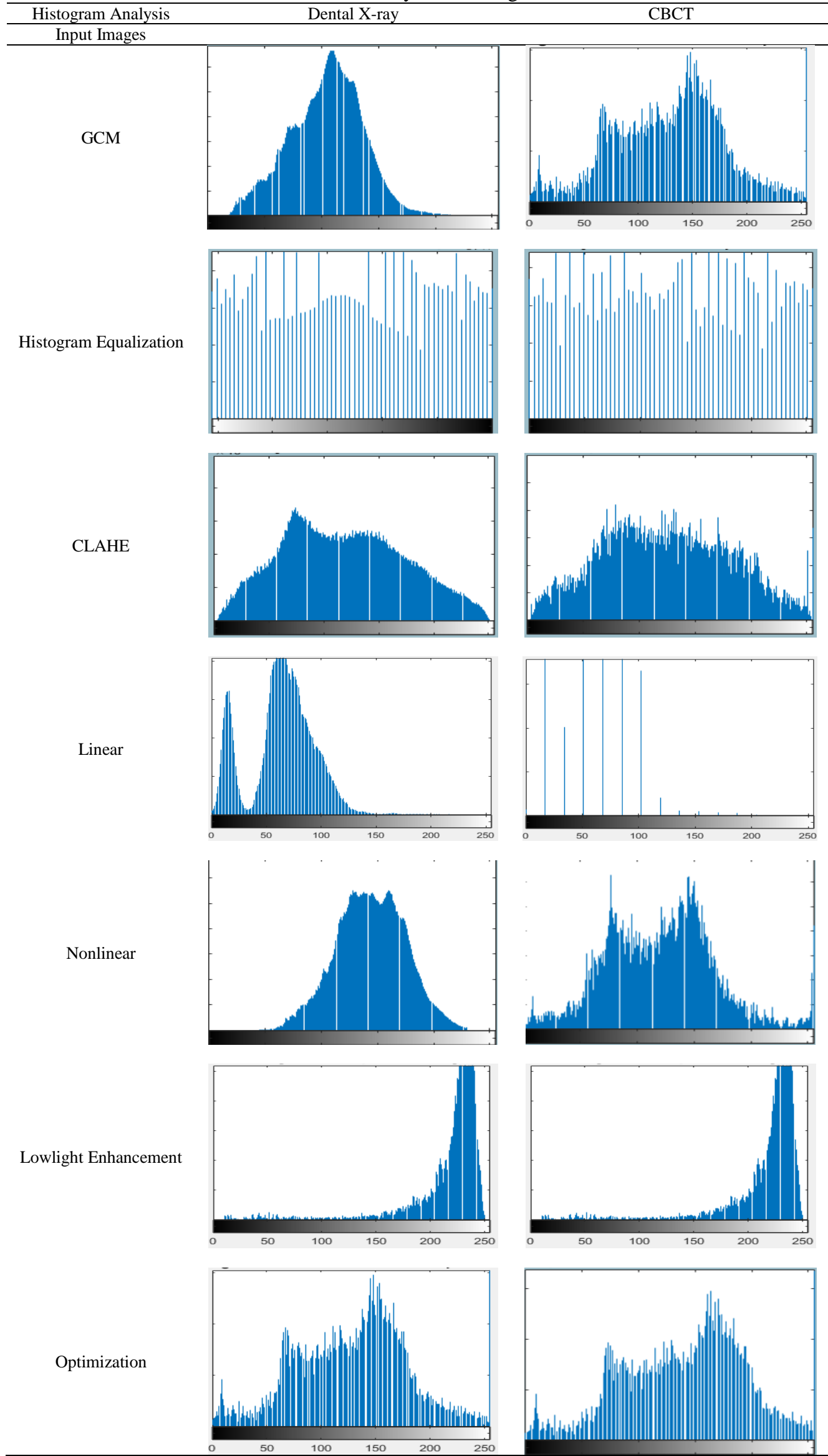

Evaluating spatial and frequency domain enhancement techniques on dental images to ... (Shashikala J.) 


\subsection{Qualitative analysis}

Table 1 demonstrates the visual outcome of the proposed framework. In this analysis, the enhancement operation is visualized for dental $\mathrm{x}$-ray and CBCT. The outcome achieved by each preprocessing technique can be evident by the human eye and visual perception. Based on the different visual perception requirements, the proposed system allows the physician to select a suitable enhancement technique. The outcome of the proposed system is also provided with the histogram analysis demonstrated in Table 2.

Table 2 highlights the qualitative analysis concerning the histogram of the images given for each input dental image and preprocessed dental images. The histogram provides the tone distribution in the image. By looking at it, the user can judge the overall tone distribution in the image. The enhanced image with better distribution of histograms reflects good and balanced contrast and brightness in the image. The analysis shows that the CLAHE, Non-linear, GCM, and histogram-based enhanced images have achieved better distribution of the contrast and brightness tone. The histogram analysis provides a better option to adjust contrast and brightness based on the requirement or the need to adjust brightness in the specific region of the image. The analysis of histogram of an image is advantageous in computer vision applications based on which complications at the implant site can be analyzed. Another beneficial factor is bone quality analysis to benefit effective and successful implant procedure.

\subsection{Quantitative analysis}

The study also presents a quantitative analysis towards identifying the effectiveness of preprocessing techniques in terms of PSNR and sharpness. Figure 3 shows the performance analysis of PSNR using GCM. The analysis is carried out for the CBCT image over progressively varying GCM values (i.e., in the range of $0.1: 1)$. It can be seen that over the increasing value of the GCM for image enhancement, the PSNR value is also increasing. However, this not means that the increased value of GCM provides higher PSNR always provides a good image. The fact is that the changing GCM leads an input image towards brighter or darker, completely based on the enhancement requirement specific to a particular input dental image. The next evaluation process is carried out for the comparative analysis of preprocessing techniques with respect to quantified PSNR and enhanced image sharpness.

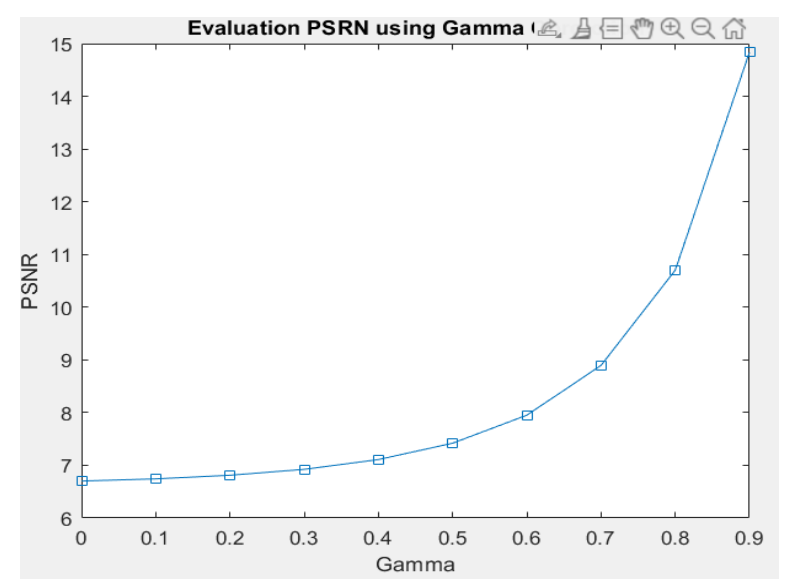

Figure 3. Analysis of PSNR over varying GCM value

In Figure 4, variation in PSNR performance is exhibited by different preprocessing techniques over a different number of input dental images. From the graph trend, it can be analyzed that the higher PSNR score is maintained by the GCM, CLAHE, optimization-based, and nonlinear enhancement techniques, respectively. However, similar PSNR performance can be seen in both CLAHE and PSO-based enhancement. The next figure presents the comparative analysis for the evaluation of image sharpness.

In Figure 5, the performance analysis is demonstrated for quantified sharpness of the enhanced images. From the graph trend, it can be seen that higher sharpness in the enhanced image is achieved using CLAHE. Also, histogram equalization, GCM, and nonlinear enhancement technique exhibited less sharpness than CLAHE, but all these techniques have achieved nearly similar performance for quantified sharpness in the output image. However, the lowlight color enhancement, linear enhancement technique, and PSO-based optimized enhancement have given less sharpness value compared to other images. 
Figure 3 and Figure 4 highlights the comparative analysis of preprocessing techniques for six different dental image enhancements. In this evaluation process, the first input image is CBCT, and the rest of the 5 input images are dental x-ray. Moreover, in the direction of conducting linear performance analysis, the value of $\gamma=0.936$ and clip limit (0.02) in the CLAHE technique is considered static for all input images. However, from both analyses, it cannot be concluded that the higher performance achieved by GCM or CLAHE is better than other techniques. This actually depends on the visual characteristics of the images. Therefore, the selection of enhancement techniques or enhanced images does not only depend on a PSNR metric. For the human visual system, the visual quality of the enhanced image is an important aspect that can show clear differences between background and objects, features in the foreground of the images. The proposed system facilitates a universal preprocessing approach that can meet multiple dental image enhancement requirements to assist in practical pre-evaluation assessment for implant therapy.

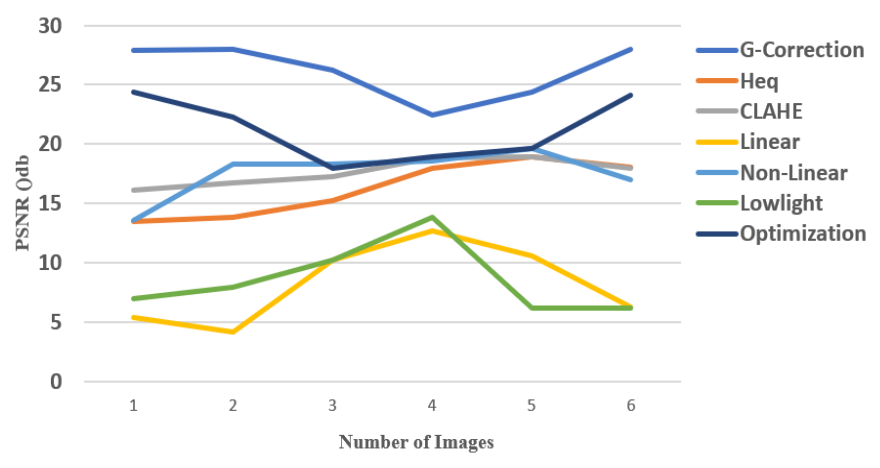

Figure 4. Analysis of PNSR for different preprocessing techniques over different images

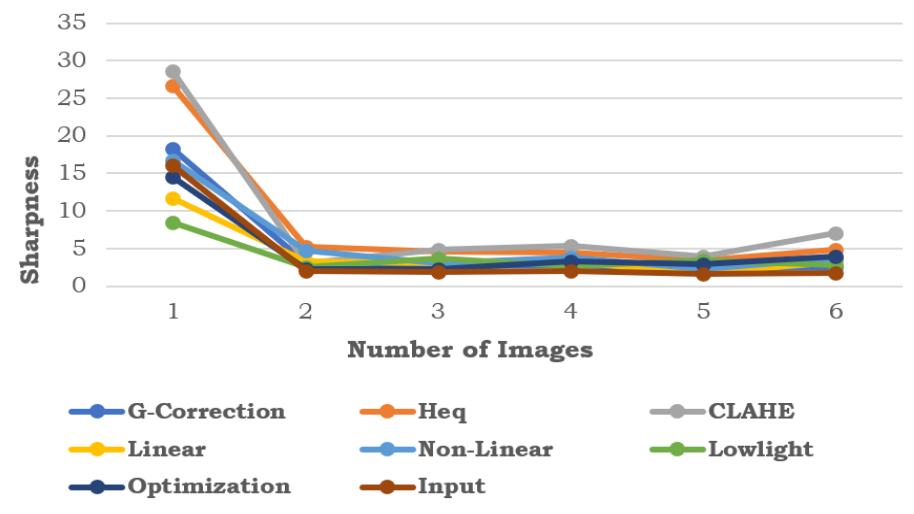

Figure 5. Analysis of PNSR for different preprocessing techniques over different input images

\section{CONCLUSION}

Choosing an appropriate preprocessing technique always a challenging task in image quality enhancement. Therefore, this paper has proposed a single-windowing scheme for carrying out effective enhancement over input images based on the integration of different image preprocessing functions. The proposed framework evaluates the effectiveness of both frequency domain enhancement and spatial domain enhancement techniques on dental images. The evaluation of preprocessing techniques was carried out for two different dental images, i.e., dental X-ray and CBCT, considering the visual outcome, PSNR, and quantified sharpness. The study outcomes show higher PSNR score is maintained by the GCM, CLAHE, optimization-based, and nonlinear enhancement technique in terms of PSNR. Higher sharpness in the enhanced image is achieved using CLAHE. However, the performance of each preprocessing depends on the visual feature of the input dental images and can be improved by fine-tuning the parameters of the preprocessing techniques. The best enhancement selection decision depends on the output image perceptibility response and statistics score. The proposed preprocessing framework offers a flexible approach to the dental expert to select an improved image of their own choice based on the visual feature of an input image and different preprocessing requirements.

Evaluating spatial and frequency domain enhancement techniques on dental images to ... (Shashikala J.) 


\section{REFERENCES}

[1] L. Lee and S. Liew, "A survey of medical image processing tools," 2015 4th International Conference on Software Engineering and Computer Systems (ICSECS), 2015, pp. 171-176, doi: 10.1109/ICSECS.2015.7333105.

[2] M. Sadrameli and M. Mupparapu, "Oral, and Maxillofacial Anatomy," Radiologic Clinics, vol. 56, no. 1, pp. 13-29, Jan. 2018, doi: 10.1016/j.rcl.2017.08.002.

[3] N. Shah, N. Bansal and A. Logani, "Recent advances in imaging technologies in dentistry," World journal of radiology, vol. 6, no. 10, pp. 794-807, Oct. 2014, doi: 10.4329/wjr.v6.i10.794.

[4] H. Rahmi-Fajrin, S. Puspita, S. Riyadi and E. Sofiani, "Dental radiography image enhancement for treatment evaluation through digital image processing," Journal of clinical and experimental dentistry, vol. 10, no. 7, pp. e629-e634, Jul. 2018, doi: 10.4317/jced.54607.

[5] D. Vijayalakshmi, M. K. Nath, and O. P. Acharya, "A Comprehensive Survey on Image Contrast Enhancement Techniques in Spatial Domain," Sensing and Imaging, vol. 21, no. 1, pp. 1-40, 2020, doi: 10.1007/s11220-02000305-3.

[6] Y.-C. Chen, Ya-Na Wu, D.-B. Shieh, C. Sun and R. R. Reisz, "3D visualization of dental anatomy in ancient fossil vertebrates by using third harmonic generation microscopy," 2014 Conference on Lasers and Electro-Optics (CLEO)-Laser Science to Photonic Applications, San Jose, CA, 2014, pp. 1-2, doi: 10.1364/CLEO_AT.2014.ATh3P.3.

[7] J. Parekh, P. Turakhia, H. Bhinderwala, and S. N. Dhage, "A Survey of Image Enhancement and Object Detection Methods," Advances in Computer, Communication and Computational Sciences, vol 1158, pp. 1035-1047, 2021, doi: 10.1007/978-981-15-4409-5_91.

[8] S. Harish and G. A. Ahammed, "Integrated modelling approach for enhancing brain MRI with flexible preprocessing capability," International Journal of Electrical and Computer Engineering (IJECE), vol. 9, no. 4, pp. 2416-2424, Aug. 2019, doi: 10.11591/ijece.v9i4.pp2416-2424.

[9] P. Vasuki, J. Kanimozhi and M. B. Devi, "A survey on image preprocessing techniques for diverse fields of medical imagery," 2017 IEEE International Conference on Electrical, Instrumentation and Communication Engineering (ICEICE), 2017, pp. 1-6, doi: 10.1109/ICEICE.2017.8192443.

[10] X. Guan, S. Jian, P. Hongda, Z. Zhiguo, and G. Haibin, "An Image Enhancement Method Based on Gamma Correction," 2009 Second International Symposium on Computational Intelligence and Design, 2009, pp. 60-63, doi: 10.1109/ISCID.2009.22.

[11] H. P. Menon and B. Rajeshwari, "Enhancement of dental digital X-ray images based on the image quality," In The International Symposium on Intelligent Systems Technologies and Applications, pp. 33-45, 2016, doi: 10.1007/9783-319-47952-1_3.

[12] S. Smriti, M. Dubey and M. I. Khan, "Comparative analysis of image enhancement techniques for ultrasound liver image," International Journal of Electrical and Computer Engineering, vol. 2, no. 6, pp. 792-797, Dec. 2012.

[13] K. Gangrade, "A Review of Various Digital Image Preprocessing Methods for Medical Image Analysis," International Journal of Computer Sciences and Engineering, vol. 7, no. 1, pp. 513-516, Jan. 2019, doi: 10.26438/ijcse/v7i1.513516.

[14] I. Scholl, T. Aach, T. M. Deserno and T. Kuhlen, "Challenges of medical image processing," Computer scienceResearch and development, vol. 26, no. 1, pp. 5-13, 2011, doi: 10.1007/s00450-010-0146-9.

[15] S. Fu, M. Zhang, C. Mu and X. Shen, "Advancements of medical image enhancement in healthcare applications," Journal of Healthcare Engineering, vol. 2018, 2018, doi: 10.1155/2018/7035264.

[16] J.-W. Choi, W.-J. Han and E.-K. Kim, "Image enhancement of digital periapical radiographs according to diagnostic tasks," Imaging Science in Dentistry, vol. 44, no. 1, pp. 31-35, 2014, doi: 10.5624/isd.2014.44.1.31.

[17] H. Kamezawa, K. Shirieda, H. Arimura, N. Kameda and M. Ohki, "An approach of exposure dose reduction of cone-beam computed tomography in an image guided patient positioning system by using various noise suppression filters," 2014 Joint 7th International Conference on Soft Computing and Intelligent Systems (SCIS) and 15th International Symposium on Advanced Intelligent Systems (ISIS), 2014, pp. 1475-1780, doi: 10.1109/SCISISIS.2014.7044706.

[18] Y. Yin, G. Yu, H. Wang, Z. Liu and D. Li, "CBCT image denoising based on multi-scale wavelet transform," 2010 3rd International Conference on Biomedical Engineering and Informatics, 2010, pp. 150-153, doi: 10.1109/BMEI.2010.5639460.

[19] S. A. Amiri and E. Moudi, "Image quality enhancement in digital panoramic radiograph," Journal of AI and Data Mining, vol. 2, no. 1, pp. 1-6, 2014, doi: 10.22044/jadm.2014.112.

[20] R. S. Kandan, A. John and S. Kumar, "An improved contrast enhancement approach for panoramic dental x-ray images," ARPN Journal Engineering Applied Science, vol. 10, pp. 1897-1901, 2015.

[21] A. Naik, S. V. Tikhe and S. D. Bhide, "Histogram Equalization for Class-Identification of Dental Disease Using Digital Radiography," in International Conference on Business Administration and Information Processing, Springer, Berlin, Heidelberg, vol. 70, pp. 144-151, 2010, doi: 10.1007/978-3-642-12214-9_25.

[22] H. M. Qassim, N. M. Basheer and M. N. Farhan, "Brightness preserving enhancement for dental digital X-ray images based on entropy and histogram analysis," Journal of Applied Science and Engineering, vol. 22, no. 1, pp. 187-94, Mar. 2019, doi: 10.6180/jase.201903_22(1).0019.

[23] T. Y. Dai and L. Zhang, "Weighted Retinex algorithm based on histogram for dental CT image enhancement," 2014 IEEE Nuclear Science Symposium and Medical Imaging Conference (NSS/MIC), 2014, pp. 1-4, doi: 10.1109/NSSMIC.2014.7430821. 
[24] V. M. Georgieva, A. D. Mihaylova and P. P. Petrov, "An application of dental X-ray image enhancement," 2017 13th International Conference on Advanced Technologies, Systems and Services in Telecommunications (TELSIKS), 2017, pp. 447-450, doi: 10.1109/TELSKS.2017.8246321.

[25] A. Khatter, A. Thakur and N. Reddy, "CBCT Image Feature Enhancement for Endodontic Therapy," 20196 th International Conference on Signal Processing and Integrated Networks (SPIN), 2019, pp. 293-296, doi: 10.1109/SPIN.2019.8711747.

[26] O. M. Gazal, A. E. Adedokun, I. J. Umoh and M. O. Momoh, "LWT-CLAHE Based Color Image Enhancement Technique: An Improved Design," Computer Engineering and Applications, vol. 9, no. 2, pp. 117-126, 2020.

\section{BIOGRAPHIES OF AUTHORS}

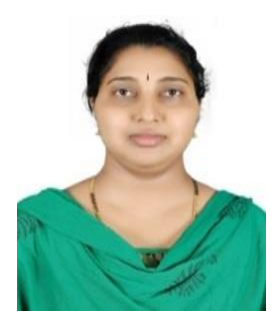

Shashikala J. is working as assistant professor in the Department of electronics and Communication at BMS institute of Technology and Management, Bengaluru, India. She has completed her completed her B. E from electronics and Communication in 2003 from UBDTCE, Kuvempu university, Davangere and M. Tech in Digital electronics and Communication in 2010 from MSRIT affiliated to VTU, Belagavi. Her areas of interest include biomedical image processing, biomedical signal processing.

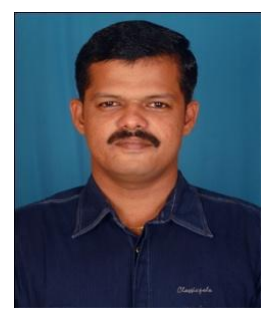

Thangadurai N. is working as Professor and Research Coordinator in the Department of Electronics and Communication Engineering, JAIN (Deemed-to-be University) Bangalore, India. He has obtained his Ph.D. Degree in Wireless Sensor Networks from Bharathiar University, Coimbatore. He has authored/co-authored 126 research papers in both International and National Journals and Conferences. He has supervised 64 numbers of undergraduate and postgraduate students for their project completion and guiding $8 \mathrm{Ph} . \mathrm{D}$., scholars now in which 2 awarded in 2019. He is currently working with sponsored research project grant received from ISRO. 